\title{
Metodología activa en la enseñanza de Odontología Legal y Forense. Experiencia en una universidad pública de Nicaragua. Enfoque cualicuantitativo
}

\author{
ALICIA ESPINOZA PALMA* \\ Universidad Nacional Autónoma de Nicaragua (UNAN-León) - Nicaragua \\ CARLOS GUEVARA ALTAMIRANO* \\ Universidad Nacional Autónoma de Nicaragua (UNAN-León) - Nicaragua \\ Recibido el 15-05-20; primera evaluación el 22-07-21; \\ segunda evaluación el 08-08-21; aceptado el 25-08-21
}

\section{RESUMEN}

Este artículo tiene la finalidad de valorar la percepción de los estudiantes en la implementación de metodologías activas para la enseñanza en Odontología Legal y Forense, desde su propia perspectiva. Se realizó un estudio de metodología mixta, tomando en cuenta 57 estudiantes que participaron en este curso y cinco informantes claves de años anteriores. La parte cualitativa se desarrolló mediante entrevistas a profundidad y análisis interpretativo reduccionista y la parte cuantitativa de tipo descriptivo. Resultados: Los estudiantes tuvieron una valoración positiva del desarrollo del componente con actividades prácticas y fue valorada como «Buena»; las expectativas al inicio del componente fueron altas y al finalizar fueron satisfechas valorado como «Bueno». El abordaje cualitativo mostró un cambio en las expectativas iniciales y finales respecto a la metodología empleada en años anteriores.

Palabras clave: percepción, estudiantes, metodología activa, odontología forense.

\footnotetext{
* Máster en Epidemiología Clínica por la Universidad Nacional Autónoma de Nicaragua-León y candidata doctoral en Ciencias Médicas, Cohorte Epidemiología y Salud Global. Secretaria académica en la Facultad de Odontología, Universidad Nacional Autónoma de Nicaragua-León. Experiencia como asistente de investigación en el Centro de Investigación en Demografía y Salud. Trayectoria docente de pregrado por once años en la UNAN-León. Profesora titular en la Facultad de Odontología. Principal de Patología Bucal I, y Odontología Legal y Forense. Docente investigadora del Departamento de Medicina Oral. https://orcid.org/0000-0002-2860-0169. Correo electrónico: samanta.espinoza@fo.unanleon.edu.ni ** Máster en Salud Pública por la Universidad Nacional Autónoma de Nicaragua-León (UNAN-León) y candidato a doctor en Educación Superior. Profesor titular en la Facultad de Odontología, Universidad Nacional Autónoma de Nicaragua-León. Docente investigador de la Facultad de Odontología. https://orcid.org/0000-0001-7130-2020. Correo electrónico: carlos.guevara@fo.unanleon.edu.ni
} 
Active methodology of teaching on Legal and Forensic Odontology. Experience in a Public University of Nicaragua. Quali-quantitative approach

\section{Abstract}

This article aims to assess student's perception in the implementation of active methodologies for teaching on Legal and Forensic Dentistry, from their own perspective. A mixed methodology study was carried out, considering 57 students who participated in this course and five key informants from previous years. The qualitative approach was developed through in-depth interviews and reductionist interpretative analysis and a descriptive study. Results: The students had a positive evaluation for course development with practical activities and it was valued as "Good"; expectations at the beginning were high and were satisfied at the end of course, valued as "Good". The qualitative approach showed a change in expectations regarding the methodology used in previous years.

Keywords: perception, students, active methodology, Forensic Odontology.

\section{Metodologia ativa no ensino de Odontologia Legal e Forense. Experiência em uma universidade pública da Nicarágua. Abordagem quali-quantitativa}

\section{Resumo}

Este artigo tem como objetivo avaliar a percepção dos alunos na implementação de metodologias ativas para o ensino de Odontologia Legal e Forense, sob sua própria perspectiva. Foi realizado um estudo de metodologia mista, levando em consideração 57 alunos que participaram deste curso e cinco informantes-chave dos anos anteriores. A parte qualitativa foi desenvolvida por meio de entrevistas aprofundadas e análise interpretativa reducionista e a parte quantitativa descritiva. Resultados: os alunos tiveram uma avaliaçáo positiva do desenvolvimento do componente com atividades práticas e foi avaliado como "Bom"; as expectativas no início do componente eram altas e no final foram satisfeitas, avaliadas como "Bom". A abordagem qualitativa mostrou uma mudança nas expectativas inicial e final em relação à metodologia utilizada nos anos anteriores.

Palavras-chave: Percepção, Alunos, Metodologias Ativas, Odontologia Forense

\section{INTRODUCCIÓN}

Las universidades públicas tienen el reto de mejorar currículos de acuerdo con niveles de exigencia del mundo globalizado. La oferta académica de la Universidad Nacional Autónoma de Nicaragua (UNAN, León) se encuentra bajo el sistema de créditos académicos desde el año 2007 y enmarcada en el modelo educativo institucional declarado constructivista, por tanto, centrado en el estudiante. Esta oferta sigue en constante revisión de sus planes para 
conservar el prestigio de esta universidad bicentenaria, que tiene planteado dar respuesta a las necesidades de la población y estar de acuerdo con los objetivos de desarrollo del milenio (Comisión de Transformación Curricular, 2019).

El cambio de modelo educativo aún no termina de establecerse en todos los componentes de la carrera de Odontología; de manera que se conservan metodologías vinculadas al modelo anterior centrado en el docente. Como parte de este proceso de cambio y asimilación es importante realizar trabajos de investigación que valoren la percepción de los estudiantes en el uso de metodologías que cambien el paradigma de enseñanza-aprendizaje en componentes como Odontología Legal y Forense. Se seleccionó este componente electivo ya que usualmente, a pesar del modelo educativo declarado, se desarrollaba a través de conferencias que tenían como fondo la transmisión de información, carente de algún protagonismo activo de los estudiantes; lo que incidió desfavorablemente en el interés por el componente a nivel de la opinática estudiantil.

A partir del año 2019, posterior a una revisión bibliográfica de los contenidos y parte de la mejora que permite el proceso de transformación curricular se realiza un cambio en la metodología de enseñanza del componente para la inclusión de actividades prácticas en la educación de Odontología Legal y Forense, que incluyeron prácticas de laboratorio, simulaciones con modelos dentales, estudio de casos de identificación ante y post mortem entre otras; (Campos et al., 2018; Hermsen y Johnson, 2012; Stamm y Palmieri, 2015), con el estudiante asumiendo la construcción de su aprendizaje; siguiendo tendencias internacionales que apoyan la enseńanza-aprendizaje en odontología forense con base en aspectos prácticos, para profundizar y resaltar la calidad e importancia de los registros dentales, craneofaciales y maximizar la aplicación dental en casos forenses(Acharya, 2006; Pereira et al., 2017). Pero, además, este cambio acerca más la metodología del componente al modelo educativo declarado por la institución, establecido como constructivista y centrado en el estudiante (Marco referencial, 2007, 2011).

Así mismo esta modificación a metodologías activas de la enseñanza se plantea desde la academia para permitir una vinculación mayor de teoría y práctica de Odontología Legal y Forense; lo que permitiría aumentar el atractivo del componente, en aras de contribuir a la formación odontológica integral.

De acuerdo con Palomares (2007) y Huber (2008) (citado por JiménezHernández, 2018) las metodologías activas permiten mayor participación, independencia del estudiantey favorecen directamentesu educación, cumpliendo una actividad más formativa que informativa, permitiendo generar experiencias 
de aprendizaje más profundos, duraderos y significativos para conseguir el desarrollo de competencias y preparan al estudiantado para el mundo laboral y asumir responsabilidades personales (Jiménez-Hernández, 2018).

Odontología Forense es la «disciplina, ciencia o especialidad que, en el ámbito médico-legal, vincula al cirujano dentista con la Ley» (Ciocca, 2010) bajo la responsabilidad ética y profesional de un registro apropiado de las características del individuo, que son parte del expediente clínico y se registran modificaciones dentales producto de tratamientos realizados. (Anadon y Robledo, 2017). Sobre todo, ante la creciente experiencia en emergencias y desastres tanto naturales como de acción humana, en que el odontólogo, como personal de salud puede realizar una contribución significativa. (Acharya, 2006; Hermsen y Johnson, 2012; More et al., 2004; Psoter et al., 2006).

La odontología forense ha sido considerada una herramientas importante en la identificación de víctimas en desastres, ya que los dientes, registros dentales diagnósticos, tratamientos o intervenciones en las estructuras orales y hasta tejidos blandos son compilados en historias clínicas, y como valor agregado tienen la utilidad de ser sistemas de comparación pre y posmortem (Blau y Briggs, 2011; Forrest, 2019; Graham, 2006). En Nicaragua la identificación de víctimas en desastres es potestad del Ministerio Público y no se tiene la especialidad en Odontología Forense como una oferta formativa en el país. Se enseńa como parte de la formación general del odontólogo, aunque el nivel de desarrollo en cuanto a la didáctica en esta disciplina es incipiente en nuestro país. Por lo que se pretende impulsar el interés en este ramo, ya que, en caso de necesidad los registros de atención dental pueden servir como evidencias para la justicia.

En cuanto al estudiante, su comportamiento académico no depende de manera absoluta de la metodología aplicada, en muchos casos es un reflejo importante de la percepción, motivación y satisfacción para su formación profesional. La satisfacción de los usuarios está enfocada desde la perspectiva de los mismos estudiantes que son los que reciben el servicio para mejorar su proceso educativo (Álvarez, Chaparro y Reyes, 2015; Sánchez, 2018) a la vez que ha cobrado más importancia de cara a la supervivencia de las instituciones públicas (Alves y Raposo citados por Álvarez, Chaparro y Reyes, 2015).

Este estudio pretende valorar la percepción de los estudiantes en la implementación de metodologías activas para la enseñanza de Odontología Legal y Forense, en una universidad pública de Nicaragua.

Preguntas de investigación:

i. ¿Cuáles fueron las expectativas de inicio y final del componente? 
ii. ¿Cuál es la percepción de los estudiantes en las actividades prácticas desarrolladas en el curso de Odontología Legal y Forense?

iii. ¿Cuál fue el nivel de satisfacción de la metodología empleada?

\section{MARCo teórico}

«La educación es un arte cuya ejecución tiene que ser perfeccionada por muchas generaciones» (Caeiro, 2009). El aprendizaje es un proceso de construcción individual y social para el estudiante (Sáenz, 2018). En el modelo educativo constructivista por competencias, el estudiante debe participar activamente como el responsable de tal construcción (Ahumada, 2005; López, 2013) y el docente, como mediador, debe proporcionar los escenarios de aprendizaje basados en la realidad y por tanto útiles (Romero-Abrio y Hurtado, 2017), que permitan al estudiante motivarse intrínseca y extrínsecamente en la construcción de su aprendizaje (Parreño, 2019; Baena, 2019).

Existen cuatro condiciones importantes para el aprendizaje efectivo (Sáenz, 2018; Peñalosa y Castańeda, 2012), de esta manera queda claro que el aprendizaje solo tiene lugar en respuesta de:

i. Las necesidades previas del estudiante relacionado a expectativas y realidades.

ii. La preparación para el aprendizaje, no solo relacionado a la estructuración de guías previas para su resolución, sino la madurez con que el estudiante logre integrar los conocimientos.

iii. Las situaciones formales de aprendizaje proporcionadas por el maestro en cuanto a un aprendizaje sistemático.

iv. El proceso de interacción y retroalimentación, que permite responder a las interrogantes que surgen en el proceso y mejora las expectativas de los estudiantes para lograr un aprendizaje efectivo.

Sumado a esto se debe considerar condiciones que favorecen el aprendizaje, tales como: a) la motivación, relacionada con la respuesta a las necesidades de los estudiantes y fundamental para un aprendizaje efectivo, en ella se conjugan la actitud del maestro y el deseo de aprender del estudiante; b) la seguridad psicológica durante la participación del estudiante, ya que debe encontrarse en un ambiente estimulante para aumentar sus posibilidades de aprendizaje. c) La experimentación como proceso activo, es una forma de contrastar la experiencia con una situación concreta. d) La retroalimentación por parte 
de estudiantes y maestros permite además una práctica de mejora e integración de conocimientos durante los procesos de evaluación; e) El sentido de pertenencia, que es resultado de la reestructuración de las experiencias obtenidas durante el proceso y f) la integración de conocimientos (Sáenz, 2018) (Collazos, Guerrero y Vergara, 2001).

Existen estudios sobre la enseñanza de Odontología Legal y Forense en diferentes países, sin embargo, pocos abordan la percepción del estudiantado, su enlace con la investigación educativa y el abordaje de metodologías activas, ninguno de ellos en nuestro país.

De la enseńanza de Odontología forense en programas de pregrado se identifica que más de la mitad de Escuelas de Odontología contienen en sus programas de 1-6 horas dedicadas a la enseńanza de Odontología Forense (Gonçalves et al., 2008; Lagos-Tissie et al., 2018; Soon et al., 2019) en algunas es de tipo obligatorio y en otras electivo (Lagos-Tissie et al., 2018).

Los métodos de enseńanza y prácticas de evaluación usualmente son conservadoras (conferencias), siendo imprescindible la práctica en la formación del futuro profesional entrenado en odontología forense (Goncalvez, Saliba, Isper y Saliba, 2008). Fueron temas comunes la identificación dental, revisión de odontología forense e identificación de víctimas en desastres. El análisis de marcas de mordida fue considerada uno de los más complejos (Manica \& Gorza, 2019).

Una evaluación de conocimientos, actitudes y prácticas de odontología forense entre estudiantes en India (Hannah et al., 2017) presenta la necesidad de profundizar y sensibilizar a los estudiantes en esta especialidad para hacerlos parte de equipos calificados en la identificación.

Estudios sobre la percepción de los estudiantes de odontología y estrategias metodológicas más satisfactorias utilizadas por sus académicos, nuestros mejores resultados al implementar aquellas, que integran conceptos teóricos y habilidades prácticas en el desarrollo de competencias (Rodriguez \& Gonzalez, 2013, Paredes y Miguel, 2017). Así también el estudiante percibe utilidad de las actividades de aprendizaje para desarrollar competencias, son valoradas positivamente y facilitan la superación de la asignatura, comportar simulación o aplicación de contenidos e implicar la interacción y discusión entre los estudiantes. (Rubio, Ruiz y Martinez-Olmo, 2016).

La satisfacción de los estudiantes con los servicios educativos brindados por Instituciones de educación Superior, hace referencia que la habilidad para la enseńanza de los docentes y el nivel de auto realización del estudiante, son variables a considerar en aquellos clasificados como mayormente satisfechos (Alvarez, Chaparro y Diana, 2014). 
Kolb, referido por Vince y Sáenz, presenta un enfoque de «las experiencias concretas o abstractas, que se transforman en conocimiento cuando reflexionamos y experimentamos». De esta manera plantea cuatro fases que son: teorizar, es seguido de experimentar, actuar y reflexionar (Vince, 1998, Sáenz, 2018).

Las metodologías activas pretenden que el estudiante sea parte activa en su aprendizaje para que este mismo sea significativo (Baena, 2019). Son métodos activos para la enseńanza la exposición grupal, la lluvia de ideas, el aprendizaje basado en problemas, método de proyectos, método de casos, entre otros (Sáenz, 2018; López-Chávez, 2016). En Odontología legal se han publicado algunas experiencias en las que se utilizan metodologías activas como las dramatizaciones y elaboración de documentos publicitarios, orientadas a la participación del odontólogo en un posible caso judicial, obteniendo como resultado la mejor asimilación de la teoría y la motivación hacia la asignatura de parte de los estudiantes (Musse, Marques, Covas y Ribeiro, 2020).

El método de proyectos es ideal en el ambiente educativo ya que permite el desarrollo de habilidades procedimentales para resolver situaciones reales y que plantean nuevas interrogantes acerca del tema. De manera que permiten mantener la motivación de estos. Los estilos de aprendizaje que favorecen son el activo, reflexivo, teórico y pragmático (Sáenz, 2018).

Los métodos de enseñanza se relacionan con la perspectiva pedagógica y constituyen un reto para docentes estructurar experiencias de aprendizaje en contexto para que el estudiante construya un aprendizaje significativo y aplicable a problemáticas reales. A continuación, abordamos algunas de ellas, que se usan en nuestro medio:

Método de conferencia/lección magistral: es un método de instrucción oral, comúnmente utilizado oralmente para el abordaje de un tema particular. Las habilidades de oratoria del maestro y su capacidad de comunicar el conocimiento son esenciales para el éxito (Sáenz, 2018). Algunas ventajas y desventajas son:

- Transmisión de gran cantidad de información en corto tiempo.

- Óptimo para grandes grupos

- Apoyo de material audiovisual

- Participación de los estudiantes reducida a preguntas

- Método centrado en el profesor

Método demostración/acción: en este se complementa la demostración del tutor, con el desarrollo de habilidades procedimentales y organizacionales de parte de los estudiantes para una aplicación práctica. La demostración se 
considera una herramienta poderosa, pues promueven confianza y certeza en el estudiante (Sáenz, 2018). Algunas de sus ventajas y desventajas son:

- Selección cuidadosa de los ejemplos o material de trabajo

- Organización adecuada del tiempo o tareas asignadas a los estudiantes

- Ensayo previo de la demostración por el personal docente

- Permite demostrar lo aprendido por los estudiantes

- Centrado en el estudiante

Método de proyectos didácticos: mejora el aprendizaje a través del compromiso y reflexión individual y colectiva de los datos presentados. Involucra la investigación y solución de problemas de la vida cotidiana a través de la integración de la teoría con la practica desde un ámbito multidisciplinario (Sáenz, 2018). Ventajas y desventajas:

- Permite la actividad y liderazgo natural en los grupos

- Involucra la interacción grupal y el debate

- Fomenta el sentido de responsabilidad

- Centrado en el estudiante

- El profesor es un facilitador

\section{Metodología}

Se realizó esta investigación con un enfoque cualicuantitativo para evaluar la percepción de los estudiantes acerca de la implementación de metodologías activas de la enseñanza del componente Odontología legal y forense, en estudiantes de licenciatura de la Facultad de Odontología, UNAN-León, en la primera cohorte expuesta al cambio referido (plan 2019).

Dentro del plan de estudios, Odontología Legal y Forense es un componente electivo de formación específica ubicado en el quinto semestre de la licenciatura en Odontología, cuenta con dos (2) créditos académicos distribuidos en dos horas presenciales por semana y es impartida por tres docentes, que apoyan a un grupo de veinte estudiantes durante las actividades prácticas (Datos no publicados, tomado de la Microprogramación del componente Plan 2019).

Requiere de conocimientos previos en diferentes disciplinas como Anatomía, Fisiología humana, Anatomía dental, Bioquímica aplicada a la Odontología, Patología general, Radiología, Patología bucal, Endodoncia, Periodoncia, Prótesis parciales, Odontopediatría y Cirugía bucal. (Datos no publicados, tomado de la Microprogramación del componente aprobado - Plan 2019). 
La evaluación del aprendizaje en este componente se realiza como parte integral del proceso educativo e incluye una evaluación inicial, formativa y sumativa, solo esta última tiene valor cuantitativo (escala de 0-100). Los estudiantes acumulan un máximo de $80 \%$ en el desarrollo de actividades prácticas y 20\% (Marco referencial, 2011) corresponde a evaluación escrita final que puede comprender preguntas de selección múltiple, presentación de casos y aplicación de mediciones o identificación en diagramas simulados.

De acuerdo con los objetivos de la investigación se desarrolló instrumento de recolección de datos que fue probado en diez estudiantes que no formaron parte de la muestra, se realizó prueba de confiabilidad ( $\alpha$ Crombach 0,89 para los 19 ítems).

La encuesta fue diseñada para este estudio utilizando preguntas de opinión que fueron valoradas en cuatro acápites, que fueron: opinión general (5 acápites), expectativas (2 acápites), percepción ( 9 acápites) y satisfacción (3 acápites). Se utilizó escala Likert, dividida en cinco categorías cuantificadas del 5 (Excelente o muy de acuerdo) al 1 (Muy mala, o nada de acuerdo, según la orientación de las preguntas realizadas). Una vez que los estudiantes estuvieron en el aula asignada para la realización del segundo examen parcial del semestre, se les solicitó llenar el instrumento de manera voluntaria y honesta según estimaran conveniente, haciendo notar que era completamente anónimo, con el objetivo de evaluar el componente.

La primera etapa, un estudio descriptivo de corte transversal para determinar la percepción de los estudiantes en tres elementos; expectativas al inicio y final del componente, percepción de las actividades desarrolladas y satisfacción de la metodología empleada.

Fueron criterios de selección para la encuesta:

- Estudiantes inscritos en el componente

- Que estuvieron presentes antes de la evaluación final del componente

- Que aceptaron participar en el estudio

En la segunda etapa se desarrolló una investigación cualitativa genérica (Percy, Kostere y Kostere, 2015) a través de entrevistas semiestructuradas y un análisis interpretativo reduccionista. La entrevista semiestructurada se realizó en cinco informantes claves, seleccionados de acuerdo a los criterios de inclusión, la entrevista se realizó de manera individual en una oficina privada de este centro de estudios sin interrupciones con un investigador experimentado; el cual tomó notas de cada respuesta y al final leyó las anotaciones para confirmar que las notas fuesen correctamente plasmadas. 
Fueron criterios de selección para los informantes claves:

- Disponibilidad para desarrollar la entrevista

- Participación en cohortes anteriores a 2019 (año del cambio de metodología)

- Disposición para expresar su opinión de manera abierta

- Promoción del componente en la primera inscripción

Para el procesamiento de la información se utilizó estadísticas descriptivas que mostraron la distribución de frecuencias y para el análisis cualitativo se utilizó el software QDA Miner versión 3.2.14 bajo el método interpretativo reduccionista o análisis cualitativo genérico, usando entrevista semiestructurada individual; seguidamente se realizó reducción de los datos a través de la identificación de códigos y categorías, los que fueron relacionados apoyados del software a través de un análisis de conglomerados. Los códigos y sus relaciones resultantes fueron esquematizados en un diagrama de causa efecto.

Aspectos éticos: No se realizó el proceso de consentimiento informado per se debido a la naturaleza de la investigación y los datos a recolectar. En cambio, se optó por informar verbalmente de las garantías de voluntariedad, anonimato, confidencialidad y uso adecuado de datos al grupo pleno; aprovechando el momento de reunión colectiva.

\section{Resultados}

\subsection{Resultados cuantitativos}

De 57 estudiantes presentados al segundo examen parcial del componente de Odontología Legal y Forense, como final del curso, se encontró que cincuenta cursaron por primera vez el componente y siete de ellos, por segunda ocasión.

Tabla 1. Percepción de utilidad del componente en estudiantes de primera y segunda inscripción.

\begin{tabular}{lcccccc}
\hline & \multicolumn{7}{c}{ ¿Cómo consideró el componente? } \\
\cline { 2 - 7 } & Nada útil & Poco útil & Poco útil & Útil & Muy útil & Total \\
\hline Primera vez & 1 & 3 & 2 & 23 & 21 & 50 \\
Segunda vez & 0 & 0 & 1 & 2 & 4 & 7 \\
Total & 1 & 3 & 3 & 25 & 25 & 57 \\
\hline
\end{tabular}


De acuerdo con la tabla 1, el componente se consideró «Muy útil» $\mathrm{y}$ «Útil» $(43,9 \%)$ en cada una de estas categorías, con porcentaje acumulado de $87,8 \%$. Las actividades fueron consideradas «Interesantes» $(47,4 \%)$ y «Muy interesantes» $(45,6 \%)$ de los casos.

$\mathrm{Al}$ abordar las expectativas, las preguntas estuvieron dirigidas en relación con expectativas al inicio del componente fueron altas y al final si fueron satisfechas. El valor medio de las expectativas al inicio del componente $(3,95)$ y al finalizar el componente $(3,82)$ con un mínimo de $(1)$ «Nada de acuerdo» y máximo de (5) «Totalmente de acuerdo». Los valores encontrados son cercanos a la categoría (4) «Muy de acuerdo» según la escala Likert.

Los resultados de la percepción de actividades prácticas realizadas en el componente según una escala de 1 al 5, donde (1) es «Muy mala» y (5) «Excelente»; fueron:

La media de percepción de las actividades prácticas fue valorada en $(4,3)$ en la categoría «Buena». De ellas la actividad mejor valorada para su aprendizaje fue la práctica de Craneometría $(4,46)$, seguido por la práctica de Diente rosado post-mortem $(4,37)$, Rugoscopia y queiloscopia $(4,30)$, siendo el seminario-taller de maltrato intrafamiliar $(4,07)$, encontrándose todas las actividades en la misma categoría.

Al valorar la Metodología del componente como apropiada para conocer la importancia de la Odontología Legal y Forense hubo 5 datos perdidos; el análisis se realizó en 52 encuestas, encontrando media de 4,27 («Bueno»). La distribución de frecuencia fue "Totalmente de acuerdo» 21 estudiantes $(36,8 \%)$ y 25 «Muy de acuerdo» (43,9\%).

$\mathrm{Al}$ preguntar, ¿Cree que lo aprendido en este componente contribuirá a su desarrollo profesional?, se encontraron "Muy de acuerdo" 25 estudiantes y 21 "Totalmente de acuerdo», para una proporción de 43,9\% y 36,8\% respectivamente. $(\mathrm{N}=56)$.

Al valorar si Vincularon y complementaron apropiadamente las actividades teóricas y prácticas; se encontró «Totalmente de acuerdo» (20) y «Muy de acuerdo" (21) para un porcentaje de $35 \%$ y $36,8 \%$, respectivamente.

En relación con la percepción de las guías desarrolladas para este fin fueron consideradas en categoría «Buenas» con media de $(4,0)$.

Respecto a la Satisfacción global en el componente de Odontología Forense, 23 estudiantes estuvieron «Muy de acuerdo» y 27 «Totalmente de acuerdo», para 40,4 y 47,4\% respectivamente. La satisfacción de los estudiantes en el desempeño del componente cursado se encontró media de 4,17, que corresponde a la categoría «Buena». 


\subsection{Resultados cualitativos}

En las notas obtenidas de las entrevistas se codificaron fragmentos significativos de texto. Se obtuvieron códigos y categorías que se relacionaron entre sí, se corroboró esta relación mediante un análisis de conglomerado jerárquico (stress 0,02 y $\mathrm{R}^{2}$ 0,91); las relaciones encontradas se esquematizan a continuación:

Figura 1. Factores involucrados en el cambio de expectativas en el componente Odontología Legal y Forense.

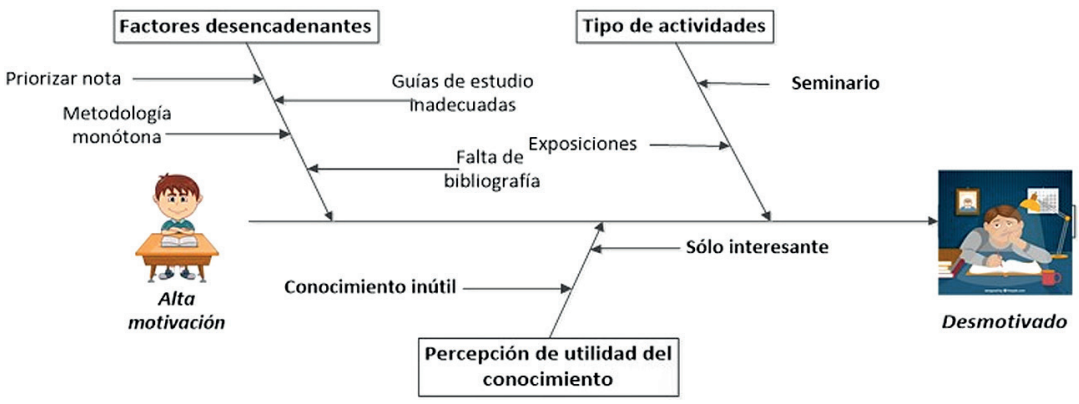

Fuente: cinco entrevistas realizadas a estudiantes de anteriores ediciones del curso.

De manera general, en lo cualitativo se encontró que existen factores como la metodología y los recursos didácticos que en conjunto con la percepción de utilidad de la asignatura y el tipo de actividades explican cómo transita un estudiante desde la motivación inicial con la asignatura hasta la desmotivación incluso con su propio desempeño académico.

En las entrevistas semiestructuradas se encontró, además, que las expectativas cambiaron al inicio y al final del curso, principalmente debido a poca aceptación de la metodología:

a mí me gustaba el nombre de la clase, porque pensé que íbamos a conocer de las leyes y el enlace con odontología... al final a mi no me gusto casi; porque era mucha teoría. (Isabel)

Según los entrevistados hubo poca satisfacción en relación a las actividades eminentemente teóricas que se desarrollaban anteriormente: «la clase era aburrida, solo nos daban un tema y nosotros teníamos que hacer las presentaciones» (Carlos); sin embargo, hay que considerar al respecto que las presentaciones, corresponden a una guía de actividades que es previamente planteada, es pues entonces una invitación a la reflexión para los tutores a mantener dinámicas que permitan la participación activa de los estudiantes. 
En este acápite, se encontró opiniones diversas, pues como lo expresa «el primer parcial fue mortal, porque casi nadie pasó» (José) y «casi no me gustó la clase» (Isabel); para ellos la referencia principal era el abordaje y la vinculación con elementos de la Odontología. Así como lo comparte «hubo bastante de las leyes de Nicaragua y del Instituto de Medicina Legal... pero no recuerdo de odontología» (Mario).

\section{Discusión}

La mayoría de estudiantes que cursaban la asignatura al momento de la encuesta valoraron el componente de manera positiva, pues las categorías "Muy útil» y «Útil», representaron el 87,8\% de las valoraciones. Así también, la mayoría consideró las actividades como «Interesantes» y «Muy interesantes» (93\%), a diferencia de lo expresado por estudiantes que cursaron el componente con la metodología anterior, para quienes el componente resultaba "Aburrido» o «Inútil» o cuando mucho «Interesante». Esto refleja un efecto directo de la metodología activa en la cual el estudiante percibe el valor del aprendizaje que él mismo construye.

En cuanto a la percepción del desarrollo del componente con actividades prácticas fue valorado por encima del punto medio de la escala (4) en la categoría «Buena», lo que coincide con el estudio «Percepción del alumnado sobre la utilidad de las actividades de aprendizaje para desarrollar competencias», donde se encontró que la mayoría de las actividades de «adquisición conceptual, simulación/ aplicación y compartir» son valoradas positivamente por el alumnado (Rubio, Ruiz y Martínez-Olmo, 2016), así como el estudio «Percepción de los estudiantes de Odontología sobre las estrategias metodológicas más satisfactorias utilizadas por sus académicos», encontró que las metodologías evaluadas con mejores resultados fueron las que integraban los conceptos teóricos y habilidades prácticas en el desarrollo de sus competencias (Fernandes, Sotolongo y Martínez, 2016; Rodríguez y González, 2013). También agregan que las estrategias metodológicas activas permiten desarrollar la capacidad analítica, autoevaluación de los estudiantes y posibilidades de desarrollar metas objetivas para el mejoramiento profesional.

Las actividades prácticas fueron incluidas a partir de 2019 en este componente y representa una primera etapa para la apropiación de conocimientos y la importancia de la evidencia en Odontología desde el ámbito Legal y Forense, lo que refleja que está de acuerdo a nuevas tendencias en educación, pues experiencias en escuelas de Odontología en países como Australia y Brasil, aun llevan a cabo la enseñanza de la Odontología Forense en sus mallas curriculares 
con métodos de enseñanza conservadoras en forma de conferencias. (Goncalvez, Saliba, Isper y Saliba, 2008) (Soon, Graham y Bassed, 2019)

De las prácticas mejor valoradas para el aprendizaje estuvieron en primer lugar Craneometría, seguido de Diente rosado posmortem, Rugoscopia y Queiloscopia, Seminario-Taller de maltrato intrafamiliar, respectivamente. Con relación a la percepción de las guías desarrolladas para este fin, fueron consideradas «Buenas», por la mayoría de los estudiantes encuestados.

Los estudiantes se expresaron satisfechos respecto a metodología del componente $80,7 \%$ ( Muy de acuerdo» y "Totalmente de acuerdo»), asimismo mostraron satisfacción sobre el aprendizaje en el componente como parte de su formación profesional. Comparable a lo registrado en el estudio «Percepción de los estudiantes de Odontología de las estrategias metodológicas más satisfactorias utilizadas por sus académicos» (Rodriguez y Gonzalez, 2013) quienes concluyen que la adquisición de habilidades fomenta el autoaprendizaje y formación continua dentro de los estudiantes, razones que hacen que se encuentren más satisfechos.

El 71,8\% de los estudiantes estuvieron satisfechos con la vinculación y complementariedad en las actividades teóricas y prácticas y el 87,8\% expresó satisfacción global en este componente.

\section{ConClusiones}

Del análisis de la encuesta de fin de curso a 57 estudiantes en el componente Odontología Legal y Forense y las entrevistas a cinco estudiantes que cursaron el componente antes del cambio de metodología, se encontraron las siguientes conclusiones

- Las expectativas al inicio del componente fueron altas y al finalizar fueron satisfechas.

- La percepción del desarrollo del componente con las actividades prácticas fue valorada en la categoría «buena».

- La satisfacción de los estudiantes en el desempeño del componente cursado se encontró en categoría «bueno».

- El cambio de metodología favorece la percepción de los estudiantes hacia el componente.

\section{RECOMENDACIONES}

- Incluir nuevas actividades prácticas en el componente Odontología Legal y Forense. 
- Mejorar guías de actividades prácticas en el componente para las próximas cohortes.

- Reforzar la importancia de los hallazgos clínicos en Odontología como evidencias desde el punto de vista Legal y Forense.

- Sistematizar la experiencia de adopción de metodologías activas de enseñanza aprendizaje.

\section{REFERENCIAS BIBLIOGRÁFICAS}

Acharya, A. (2006). Teaching forensic odontology: An opinion on its content and format. European Journal of Dental Education, 10(3), 137-141. https://doi. org/10.1111/j.1600-0579.2006.00405.x

Ahumada, P. (2005). La evaluación auténtica: un sistema para la obtención de evidencias y vivencias de los aprendizajes. Perspectiva Educacional, Formación de profesores, (45), 11-24.

Álvarez, J., Chaparro, E. y Reyes, D. (2015). Estudio de la Satisfacción de los Estudiantes con los servicios Educativos brindados por instituciones de Educación Superior del Valle de Toluca. Revista Iberoamericana sobre Calidad, Eficacia y Cambio en Educación, 5-26.

Anadon, M. y Robledo, M. (2017). Manual de Criminalística y Ciencias Forenses. En M. Anadon y M. Robledo, Manual de Criminalistica y Ciencias Forenses. Madrid, España: Editorial Tébar Flores.

Baena, A. (2019). Metodologías activas en ciencias de la educación. Vol. 1. Sevilla: Wanceulen editorial. https://doi.org/10.33996/revistahorizontes.v1i1.23

Blau, S. y Briggs, C. A. (2011). The role of forensic anthropology in Disaster Victim Identification (DVI). Forensic Science International, 205(1-3), 29-35. https://doi.org/10.1016/j.forsciint.2010.07.038

Caeiro, O. (2009). Sobre pedagogía. Córdoba: Brujas.

Campos, L. C., Contreras, D. S., Daza, S. A. y del Pilar Pedraza, A. (2018). Revisión sistemática de rugoscopia en odontología forense como técnica de identificación de personas. Ustasalud, 17(1-S), 74.

Ciocca, L. (2010). Odontología Médico Legal: aspectos forenses, profesionales y sociales. En L. C. Gómez, Odontología médico legal: aspectos forenses, profesionales y sociales. Santiago, Chile: Eds. Jurídicas de Santiago.

Collazos, C., Guerrero, L. y Vergara, A. (2001). Aprendizaje Colaborativo: un cambio en el rol del profesor. En Proceedings of the 3rd Workshop on Education on Computing.

Comisión de Transformación Curricular. (2019). Macroprogamación de la Carrera de Odontología. León: Facultad de Odontología. 
Fernandes, D., Sotolongo, M. y Martínez, C. (2016). La evaluación del desempeño por competencias: percepciones de docentes y estudiantes en la Educación Superior. Formación Universitaria, 15-24. https://doi.org/10.4067/ S0718-50062016000500003

Forrest, A. (2019). Forensic odontology in DVI: Current practice and recent advances. Forensic Sciences Research, 4(4), 316-330. https://doi.org/10.108 0/20961790.2019.1678710

Goncalvez, P., Saliba, C., Isper, A. y Saliba, N. (2008). The teaching of Deontology andlor Legal Dentistry on Brazilian Dental. São Paulo: Programa de Postgrado en Odontología Preventiva y Social NEPESCO.

Graham, E. A. (2006). Disaster victim identification. Forensic Science, Medicine, and Pathology, 2(3), 203-207. https://doi.org/10.1007/s12024-006-0011-0

Hannah, R., Ramani, P., Natesan, A., Sherlin, H. J., Gheena, S., Ramasubramanian, A., Jayaraj, G., Don, K. y Archana, S. (2017). Evaluation of knowledge, attitude and practice of forensic odontology among undergraduate dental students. International Journal of Orofacial Biology, 1(1), 16.

Hermsen, K. P. y Johnson, J. D. (2012). A model for forensic dental education in the predoctoral dental school curriculum. Journal of Dental Education, 76(5), 553-561. https://doi.org/10.1002/j.0022-0337.2012.76.5.tb05289.x

Jiménez-Hernández, D. (2018). Métodos didácticos activos en el sistema universitario actual. En D. Jiménez-Hernández, Métodos didácticos activos en el sistema universitario actual (p. 50). Murcia: Dykinson. https://doi. org/10.2307/j.ctv346q0p

Lagos-Tissie, D., Bravo, L., Ramos-Moreno, J. L. y Ciocca-Gómez, L. (2018). Docencia e investigación en Odontología Legal en Chile: situación y desafíos actuales. International Journal of Odontostomatology, 12(3), 274-279. https://doi.org/10.4067/S0718-381X2018000300274

López, F. (2013). Metodología participativa en la enseñanza universitaria. Madrid: Narcea Ediciones.

López-Chávez, V. (2016). Tipos de aprendizaje, características y principios de un aprendizaje efectivo. Vida Científica Boletín Cientifico De La Escuela Preparatoria No. 4, 4(8). Recuperado de https://repository.uaeh.edu.mx/ revistas/index.php/prepa4/article/view/422

Manica, S. \& Gorza, L. (2019). Forensic Odontology in the 21st Centuryidentifying the opinions of those behind the teaching. Journal of Forensic and Legal Medicine, 64, 7-13. https://doi.org/10.1016/j.jflm.2019.03.006

More, F. G., Phelan, J., Boylan, R., Glotzer, D., Psoter, W., Robbins, M., Rekow, E. D. y Alfano, M. C. (2004). Predoctoral dental school curriculum for catastrophe preparedness. Journal of Dental Education, 68(8), 851-858. https://doi.org/10.1002/j.0022-0337.2004.68.8.tb03834.x 
Musse, J. O., Marques, J. A. M., Covas, A. K. O. y Ribeiro, M. S. (2020). Metodologias ativas de ensino em Odontologia Legal. REVISA, 9(3), 524-528. https://doi.org/10.36239/revisa.v9.n3.p524a528

Paredes, M., y Miguel, O. (2017, marzo 3). Percepción de calidad de los estudiantes de 4 to y 5 to año de los programas de la carrera de Odontología de la UNAH, Tegucigalpa. Managua: CIES.

Parreño, C. M. T. (2019). El constructivismo, según bases teóricas de César Coll. Revista Andina de Educación, 2(1), 25-28. https://doi.org/10.32719/2631 2816.2019.2.1.4

Peñalosa, E., y Castañeda, S. (2012). Identificación de predictores para el aprendizaje efectivo en línea: un modelo de ecuaciones estructurales. Revista Mexicana de Investigación Educativa, 247-285.

Percy, W. H., Kostere, K. y Kostere, S. (2015). Generic qualitative research in psychology. The Qualitative Report, 20(2), 76-85. https://doi.org/10. 46743/2160-3715/2015.2097

Pereira, J. G. D., Recalde, T. S. F., Costa, P. B., Jacometti, V., Magalhães, L. V. y Da Silva, R. H. A. (2017). Forensic odontology education: From undergraduate to $\mathrm{PhD}$-a Brazilian experience. The Journal of Forensic OdontoStomatology, 35(2), 149.

Rodríguez, M. P. y González, S. (2013). Percepción de los estudiantes de Odontología de las estrategias metodológicas más satisfactorias utilizadas por sus académicos. Revista de Educación en Ciencias de la Salud, 42-46.

Romero-Abrio, A. y Hurtado, S. (2017). ¿Hacia dónde va el rol del docente en el siglo XXI? Estudio comparativo de casos reales basados en las teorías constructivista y conectivista. Hekademos: Revista Educativa Digital, (22), 84-92.

Rubio, M., Ruiz, A. y Martínez-Olmo, F. (2016). Percepción del alumnado sobre la utilidad de las actividades de aprendizaje para desarrollar competencias. Revista de Investigación Educativa, 221-240. https://doi.org/10.6018/ rie.34.1.225131

Stamm, A. D. B. y Palmieri, J. E. (2015). Diente Rosado Postmortem y Odontología Forense: Relato de Caso Pericial. Revista Skopein, 10.

Sáenz, J. (2018). Estilos de aprendizaje y métodos de enseñanza. Madrid: UNED.

Sánchez, J. (2018). Satisfacción estudiantil en educación superior. En J. Sánchez, Satisfacción estudiantil en educación superior: validez de su medición (pp. 1-37). Bogotá, Colombia: Universidad Sergio Arboleda.

Soon, A., Graham, J. y Bassed, R. (2019). Teaching of forensic Odontology in basic dental program in nine Australian dental School: A survey. Eur J Dent Educ, 244-250. https://doi.org/10.1111/eje.12425 
Vince, R. (1998). Behind and Beyond Kolb's Learning Cycle. Journal of Management Education, 304-319. https://doi.org/10.1177/105256299802200304

\section{ANEXO}

\section{Instrumento de recolección de datos (encuesta)}

Estimado estudiante, estamos finalizando el componente de Odontología Legal y Forense, 2019. Favor llenar de manera consciente cada uno de los acápites que se presentan a continuación para la valoración de la metodología empleada. Siéntase libre de escribir su valoración pues es completamente ANÓNIMA. Sus datos permitirán mejorar nuestro desempeño. (Marque con *)

\begin{tabular}{|c|c|c|c|c|}
\hline $\begin{array}{l}\text { Año en el que está inscrito } \\
3^{\circ}-\end{array}$ & \multicolumn{2}{|c|}{$\begin{array}{l}\text { Ha llevado el componente } \\
\text { Primera vez__ } \\
\text { Segunda vez__ } \\
\text { Tercera vez__ }\end{array}$} & $\begin{array}{l}\text { Consideró e } \\
\text { Muy útil__ } \\
\text { Indiferente } \\
\text { Nada útil }\end{array}$ & $\begin{array}{l}\text { Ótil } \\
\text { Poco útil }\end{array}$ \\
\hline \multicolumn{2}{|c|}{$\begin{array}{l}\text { Las actividades las consideró: } \\
\text { Muy interesantes___ Interesantes __ } \\
\text { Indiferentes___ }\end{array}$} & \multicolumn{3}{|c|}{$\begin{array}{l}\text { Su motivación en el componente fue: } \\
\text { Alta__ Moderada__ } \\
\text { Regular__ Nula__ }\end{array}$} \\
\hline
\end{tabular}

\begin{tabular}{|c|c|c|c|c|c|}
\hline & \multicolumn{5}{|c|}{ ESCALA DE LIKERT } \\
\hline EXPECTATIVAS & $\begin{array}{l}\text { TOTALMENTE } \\
\text { DE ACUERDO }\end{array}$ & $\begin{array}{l}\text { MUY DE } \\
\text { ACUERDO }\end{array}$ & INDECISO & $\begin{array}{l}\text { POCO DE } \\
\text { ACUERDO }\end{array}$ & $\begin{array}{l}\text { NADA DE } \\
\text { ACUERDO }\end{array}$ \\
\hline $\begin{array}{l}\text { Sus expectativas al } \\
\text { inicio del componente } \\
\text { fueron altas }\end{array}$ & & & & & \\
\hline $\begin{array}{l}\text { Sus expectativas al } \\
\text { finalizar el componente } \\
\text { fueron satisfechas }\end{array}$ & & & & & \\
\hline PERCEPCIÓN & EXCELENTE & BUENA & REGULAR & MALA & $\begin{array}{l}\text { MUY } \\
\text { MALA }\end{array}$ \\
\hline $\begin{array}{l}\text { ¿Cómo consideró el } \\
\text { laboratorio de diente } \\
\text { rosado post-mortem } \\
\text { para su aprendizaje? }\end{array}$ & & & & & \\
\hline $\begin{array}{l}\text { ¿Cómo considero la } \\
\text { guía para su desarrollo? }\end{array}$ & & & & & \\
\hline
\end{tabular}




\begin{tabular}{|c|c|c|c|c|c|}
\hline \multicolumn{6}{|l|}{$\begin{array}{l}\text { ¿Cómo consideró la } \\
\text { práctica de rugoscopia } \\
\text { y queiloscopia para su } \\
\text { aprendizaje? }\end{array}$} \\
\hline $\begin{array}{l}\text { ¿Cómo consideró la } \\
\text { guía para su desarrollo? }\end{array}$ & & & & & \\
\hline $\begin{array}{l}\text { ¿Cómo consideró la } \\
\text { práctica de craneo- } \\
\text { metría para su apren- } \\
\text { dizaje? }\end{array}$ & & & & & \\
\hline $\begin{array}{l}\text { ¿Cómo consideró la } \\
\text { guía para su desarrollo? }\end{array}$ & & & & & \\
\hline $\begin{array}{l}\text { ¿Cómo consideró el } \\
\text { seminario taller maltra- } \\
\text { to intrafamiliar para su } \\
\text { aprendizaje? }\end{array}$ & & & & & \\
\hline $\begin{array}{l}\text { ¿Cómo consideró la } \\
\text { guía para su desarrollo? }\end{array}$ & & & & & \\
\hline $\begin{array}{l}\text { ¿Cómo fue su expe- } \\
\text { riencia global en el } \\
\text { componente de Odon- } \\
\text { tología Legal y Forense } \\
2019 \text { ? }\end{array}$ & & & & & \\
\hline SATISFACCIÓN & $\begin{array}{l}\text { TOTALMENTE } \\
\text { DE ACUERDO }\end{array}$ & $\begin{array}{l}\text { MUY DE } \\
\text { ACUERDO }\end{array}$ & INDECISO & $\begin{array}{l}\text { POCO DE } \\
\text { ACUERDO }\end{array}$ & $\begin{array}{l}\text { NADA DE } \\
\text { ACUERDO }\end{array}$ \\
\hline $\begin{array}{l}\text { Cree que la metodolo- } \\
\text { gía del componente es } \\
\text { apropiada para conocer } \\
\text { la importancia de la } \\
\text { Odontología Legal y } \\
\text { Forense }\end{array}$ & & & & & \\
\hline $\begin{array}{l}\text { Cree usted que lo } \\
\text { aprendido en este } \\
\text { componente contri- } \\
\text { buirá a su desarrollo } \\
\text { profesional }\end{array}$ & & & & & \\
\hline $\begin{array}{l}\text { Se vincularon y com- } \\
\text { plementaron apropia- } \\
\text { damente las actividades } \\
\text { teóricas y prácticas }\end{array}$ & & & & & \\
\hline
\end{tabular}


Alicia Espinoza Palma y Carlos Guevara Altamirano

Guia de entrevista semiestructurada

1. ¿Cómo describirías tu experiencia en el componente Odontología legal y forense?

2. ¿Cuál es tu opinión acerca de los aspectos metodológicos del componente? 